Catherine Khordoc

Comment ? Lamentations essayistiques chez Monique Bosco 


\title{
Résumé
}

Cet article entreprend une analyse des essais de Monique Bosco, publiés pendant les huit dernières années de sa vie, entre 1998 et 2006, à la lumière de la tradition des lamentations. En examinant le recours à l'intertextualité dans ces essais, notre étude privilégie la dimension de la lamentation qui vise l'interrogation plutôt que la complainte. Dans le contexte de la Shoah, Bosco cherche, à l'instar du Livre des Lamentations, à comprendre comment ce qui est arrivé a pu avoir lieu, une quête qui passe nécessairement par la lecture de textes historiques, philosophiques et autobiographiques.

\begin{abstract}
This article undertakes an analysis of Monique Bosco's essays, published during the last eight years of her life, between 1998 and 2006, in light of the lament tradition. Focusing on the use of intertextuality in these essays, the article emphasizes lamentation as an interrogation rather than a lament, a notion that perhaps comes more immediately to mind. In the context of the Shoah, Bosco seeks to understand in the manner of the Book of Lamentations how what happened could have happened, a quest that necessarily involves the reading of historical, philosophical and autobiographical texts.
\end{abstract}

Oui, de toutes mes forces, forcément déclinantes, je veux comprendre et parler, et parler encore, et expliquer, et chercher, rechercher, fouiller à en avoir les mains en sang et les yeux brûlés, je veux désenfouir et faire enfin venir au jour l'affreux passé, mon passé et celui des miens qui ne sont plus là pour m'aider.

Lors d'un entretien avec l'éminent critique littéraire québécois André Brochu, Monique Bosco affirme que son écriture est motivée par la lamentation. En réponse à la question de Brochu, qui lui demande si la lamentation ne pourrait pas être considérée comme étant un « véritable genre littéraire, » elle admet que pour elle, «"faire le mur, [...] cela voulait exactement dire cela : me lamenter, avec abondance, pendant des heures. Et je n'étais jamais à court d'inspiration. ${ }^{2}$ Certes, il n'y a aucune pénurie de malheurs dans le monde pour nourrir les lamentations de Bosco : guerres, pogroms, génocides, complots politiques, migrations forcées, haine de l'autre, désastres naturels, que Bosco ne se prive pas d'évoquer dans son œuvre. Les œuvres de fiction et de non-fiction de cette autrice québécoise issue d'une famille juive d'origine autrichienne manifestent une préoccupation incontournable envers tout ce qui touche à la perte, au malheur, au désespoir, à l'injustice et aux préjugés. Ce n'est donc pas étonnant à ce que la Shoah soit inscrite dans l'œuvre de Bosco, que ce soit dans les 
poèmes, les nouvelles, les romans, ou encore, les essais, provoquant l'impulsion de se lamenter. Mais si Bosco fait allusion au génocide juif assez fréquemment, elle le fait de manière évasive, suggérant qu'elle hésite à entrer de plain-pied dans les affres de ce sombre épisode de l'histoire contemporaine qui est aussi celle des siens. Elle admet en outre, dans ses essais que sa mémoire est en jeu, son instabilité ne lui permettant pas de saisir pleinement le degré de pertes et de souffrances éprouvées par les membres de sa famille étendue. L'oscillation entre mémoire et oubli rythme ses textes de manière qui rappelle d'ailleurs cette tradition de la lamentation dont Bosco se revendique.

Or, la lamentation qu'entreprend Bosco ne se limite pas à une complainte pour les millions de morts. Dans ses textes, le sujet, qu'il soit poétique, narratif ou essayistique, veut surtout comprendre comment ce qui relève de l'impossibilité, l'inimaginable, l'inconcevable a pu se produire. Pourquoi n'a-t-il pas été possible de prévoir ce que fomentait Hitler et d'empêcher les atrocités commises en Europe pendant la Seconde Guerre mondiale? Comment expliquer la haine et la méfiance envers les Juifs? Pour Bosco, comme pour tant d'autres, la Shoah représente le «chaos originel, [le] chaos de notre époque " mettant à l'épreuve toute possibilité de compréhension, de raisonnement. «Mais comment survivre à cela, » se demande-t-elle, même en ce qui concerne ceux qui y ont, en effet, survécu. ${ }^{3}$

La tradition de la lamentation ne s'apparente pas exclusivement au chant ou au ressassement pour pleurer les êtres disparus et les malheurs. Si le Livre des Lamentations exprime certes la profonde douleur provoquée par la destruction de Jérusalem, ce texte entreprend également une quête d'explication de ce ravage causé par l'incompréhensible volonté de Dieu. Le tout premier mot du texte biblique est comment. C'est à Dieu que l'auteur des Lamentations posait la question, à savoir comment Dieu eut pu permettre la destruction de Jérusalem et la perte de vies innocentes. Pourquoi un jugement si sévère envers tout un peuple, même s'il avait négligé de respecter certaines lois ou avait péché ? Dans le préambule de sa traduction du Livre des Lamentations, Jean-Marc Droin explique que le texte biblique outrepasse le cri de douleur exprimant la souffrance insoutenable provoquée par la destruction de Jérusalem : «Comment? Comment cette catastrophe? Comment est-il possible que Dieu laisse faire, se taise ? ${ }^{4}$

En effet, ce questionnement n'est pas étranger à l'égard de la Shoah. Dans son étude de la «littérature de la survie,» Tod Linafelt affirme que l'étude de la Shoah est particulièrement pertinente pour l'étude de l'histoire de l'interprétation du Livre des Lamentations. ${ }^{5}$ Dans Babel-Opéra, texte de Bosco qui chevauche fiction, autobiographie et poésie, la voix narrative interroge directement Dieu, employant la deuxième personne du singulier, l'interrogeant à propos de sa négligence en regard de la Shoah : «Il a bien fallu que tu sois aveugle, durant des années, pour laisser arriver 
ce que jamais on ne vit, auparavant. [...] Tu n'as rien vu, apparemment et pendant toutes ces années. [...] Comment croire que tu as pu t'absenter, si longtemps, insensible à l'holocauste. ${ }^{6}$ Ce type de questionnement se poursuit dans les essais de Bosco, même lorsqu'elle ne s'adresse plus à Dieu. Comme le souligne Leora Batnitzky, il faut faire la différence entre accusation et lamentation; contrairement au premier terme, la lamentation n'attend pas de réponse ou d'explication, voire il n'en existe aucune.7 Si, dans ses écrits, Bosco cherche à comprendre, elle ne s'attend pas à obtenir de réponse, surtout en ce qui a trait à la Shoah, qui demeure insaisissable et incompréhensible.

Dans son essai Ninfa dolorosa : Essai sur la mémoire d'un geste dans lequel il aborde les échanges entre Walter Benjamin et Gershom Scholem, Georges Didi-Huberman examine l'expression et la représentation de la souffrance dans l'art contemporain. Parmi les orientations qu'il dégage des écrits de Scholem, il y en a deux qui sont particulièrement éclairantes pour notre approche à l'œuvre de Bosco. Soit, se lamenter, «c'est questionner, de la façon la plus littérale (philologiquement) et la plus radicale (philosophiquement) qui soit » et «se lamenter, c'est scander. C'est répéter en boucles multiples qui se transforment éventuellement. C'est faire haleter les phrases que l'on prononce. C'est rythmer, c'est changer déjà. ${ }^{8}$ C'est donc dans cette double optique du questionnement et de la répétition textuelle que nous examinons les essais de Bosco. La citation qui se trouve en exergue incarne ces deux fils de la lamentation qui nous intéressent, soit les effets de répétitions qui rythment le texte rappelant le chant et la complainte d'une part, et d'autre part, l'ardent désir de comprendre même s'il s'avère inassouvissable.

Née en 1927 à Vienne, Monique Bosco a grandi en France, d'abord à Paris et plus tard, lorsque la Seconde Guerre mondiale a éclaté, à Marseille. Elle a vécu une partie de cette période, à l'adolescence, cachée et privée de ses classes au lycée. Grande lectrice, elle entreprendra des études en lettres à l'Université de Montréal peu de temps après avoir atterri au Québec en 1948, où elle rédigera une thèse de doctorat sur le thème de la solitude dans la littérature canadienne-française - cela à une époque où il était plus courant de faire un doctorat en France, et de surcroit, relativement rare de se pencher sur la littérature canadienne-française. Après avoir pratiqué le métier de journaliste, elle obtiendra en 1963 un poste de professeure de littérature à cette même université où elle contribuera au programme en création littéraire. Bosco a publié une vingtaine de livres : romans, recueils de nouvelles et de poésie, essais, ainsi que quelques textes épars dans des revues québécoises, incluant une pièce de théâtre et de courts textes personnels, sur une période qui s'étend sur six décennies, soit entre 1960 et 2006. Son dernier essai, Ces gens-là, ${ }^{9}$ parait à peine un an avant son décès en 2007 . 
Les œuvres de Bosco n'hésitent pas à exprimer la facette de la lamentation qui vient peut-être le plus spontanément à l'esprit, à savoir le tourment, le désarroi, la peine incommensurable, qu'engendrent la méfiance et la haine envers les Juifs depuis des siècles, concrétisées entre autres par les pogroms du dix-neuvième siècle et ultimement par la Shoah. Dans son étude sur La femme de Loth, troisième roman de Bosco publié en 1970, Nancy Arenberg soutient que la souffrance qui y est exprimée se transforme en «lamentation textuelle,» rappelant d'ailleurs que ce rituel est généralement associé aux femmes dans la Bible..$^{10}$ En outre, Bosco fait référence explicitement à la lamentation dès l'exergue de son premier roman, Un amour maladroit," ce qui mène Michael Greenstein à constater : "In these lines Bosco announced her obsession with lamentation : repetitive tears in front of repetitive walls that connect her to her ancestors. $»^{12}$ Or, dans son analyse de ce même roman, Irène Oore signale que le «Mur,» qu'il serait plus juste d'appeler le «Mur occidental,» plutôt que le «Mur des lamentations, » n'est pas un lieu de lamentations, mais bien de prières..$^{13}$ Mais il reste que l'allusion à la lamentation dans cette œuvre demeure tout à fait pertinente, toujours selon Oore : «Au niveau littéral, le texte liminaire constitue une expression de douleur, de désarroi et d'impuissance, expression que le "Mur des lamentations" semble inviter. Il s'agit d'un chant constitué de répétitions multiples ("mur" est évoqué quatre fois; "lamentation," trois fois) et au rythme incantatoire. Le roman sera justement un tel chant. $»^{\text {I4 }}$ Par rapport à une nouvelle de Bosco, dont le titre est particulièrement à propos, «Les lamentations de la vieille en ce jour du Kippour, $»^{15}$ Cristina Minelle relève les différences formelles entre le genre de la nouvelle et celui des lamentations, qu'elle associe non seulement à la complainte, mais aussi au repentir et à l'expiation. ${ }^{16}$ En effet, il est plutôt question dans cette nouvelle du jour du Kippour qu'aux Lamentations, mais ce titre, à l'instar de l'exergue d'Un amour maladroit, incite à une réflexion sur les lamentations. Cependant, si ces études constatent le désir des protagonistes à vouloir comprendre, elles ne relèvent pas le lien entre questionnement et lamentation. Les études qui font le lien entre les lamentations et les écrits de Bosco privilégient la dimension «complainte » ou «pleurs" plutôt que celui entre lamentation et interrogation.

S'il y a beaucoup à dire à propos de la fiction de Bosco, comme les articles susmentionnés l'illustrent, ainsi que d'autres études portant sur l'inscription des mythes, le thème de la mémoire et les quêtes identitaires, entre autres, ${ }_{17}^{17}$ c'est surtout aux essais que s'attarde cet article. Plus précisément, c'est le recours à l'intertextualité, à la fois véhicule de tentative de mieux comprendre et stratégie de répétition, qui est au centre de notre propos. Comme les œuvres de fiction, les essais participent à ce que nous nommons une poétique de lamentation. Cependant, cette poétique s'y déploie de manière distinctive, le questionnement étant particulièrement explicite et, de surcroit, inextricablement lié aux préoccupations sociales et historiques auxquelles Bosco réfléchit. Justement, ce qui la désole au plus haut point c'est l'impression que personne n'a voulu vraiment savoir, n'a cherché à vraiment comprendre, ce 
qui se passait - que ce soit à propos de la Shoah, des pogroms, d'autres génocides ou conflits armés. Le danger est d'autant plus grave lorsque l'ignorance est arrimée à l'oubli et, pour le contrer, il faut lire et remplir son devoir de mémoire. Voilà les deux fils que Bosco tisse afin de pouvoir exercer ce qu'elle nomme son «devoir de vigilance. $\aleph^{18} \mathrm{Si}$, pour Bosco, l'écriture constitue une nécessité, c'est avant tout la lecture qui pourrait constituer une arme de défense, dans la quête de la compréhension et la lutte contre les injustices, quelles qu'elles soient.

Personne ne cherche plus à lire, vraiment à lire, avec l'attention nécessaire pour essayer de comprendre ce qui se trame à notre insu. Là serait notre rôle, à nous les anciens profs prématurément mis à la retraite : répéter avec vigilance que rien ne remplacera l'art de lire et de déchiffrer des textes; que seules l'écriture et la lecture nous permettront de demeurer des adultes raisonnables et responsables, de comprendre avant qu'il ne soit trop tard ce qu'il faut connaître et analyser dans le nouveau monde où nous vivons tant bien que mal. ${ }^{19}$

Les lectures de Bosco sont motivées par le désir inassouvissable de comprendre : comprendre comment les nazis ont réussi non seulement à déclencher la Seconde Guerre mondiale, mais à mener une campagne génocidaire sans vergogne; comprendre la haine inexplicable envers les Juifs; comprendre pourquoi les écrits précurseurs des philosophes et d'autres penseurs n'ont pas été davantage lus et entendus; pourquoi même à l'époque contemporaine, c'est-à-dire au début du vingt-et-unième siècle, nous continuons à être ignorants et à fermer les yeux sur la haine et le mépris les uns envers les autres. Pour Bosco, ce sont les livres qui présentent les meilleurs outils menant à un début de compréhension. Elle parle des livres comme étant des «antidotes $»^{20}$ où l'on peut trouver des éclaircissements voire des « réponses. $»^{21}$

Le passage précité illustre de surcroit l'importance de la répétition, rappelant le rituel performatif de la lamentation. Effectivement, de nombreux effets de répétition traversent ces lamentations textuelles, contribuant ainsi à rythmer les textes de Bosco, que ce soit par le truchement des citations, de références réitérées à certains auteurs de prédilection, de certaines phrases reprises mot pour mot ou de refrains de chansons connues..$^{22}$ Élisabeth Nardout-Lafarge fait remarquer que la confession que Bosco entreprend dans ses essais fonctionne justement «comme un performatif, fortement ritualisé, et quasi intransitif. ${ }^{23}$ La volonté de se confesser, alignée à d'autres dimensions telles que les nombreuses lectures commentées et le discours sur la mémoire s'enchainent pour constituer ces lamentations de Bosco.

\section{Interrogations intertextuelles}

Dans le Livre des Lamentations, c'est surtout à Dieu que l'auteur ${ }^{24}$ demande des explications. Si Bosco s'adresse parfois à Dieu, ce n'est pas de lui qu'elle s'attend à recevoir 
des explications. S'éloignant du ton accusateur qui surgit parfois, ${ }^{25}$ Bosco se tourne plutôt vers des livres écrits par des penseurs qui ont eux-mêmes souffert ou qui se sont penchés sur les questions de la souffrance, de l'injustice et de la Shoah, entre autres, pour tenter de mieux comprendre l'état du monde, l'émergence de conflits et les contextes de discrimination systémique. Lectrice insatiable, Bosco cite et commente abondamment ses lectures, tissant des liens entre écrivains, œuvres, lectures et événements, manifestation évidente de l'ampleur de sa réflexion et de l'acharnement avec lequel elle se consacre à son questionnement. Elle ne cesse de lire et ses choix de lectures ne connaissent pas de limites. Bosco se tourne vers des œuvres de fiction (À la recherche du temps perdu de Proust, Guerre de Timothy Findlay, Les liaisons dangereuses de Choderlos Laclos, Mrs. Dalloway de Virginia Woolf, Agua Viva de Clarice Lispector, $L a$ Tache de Philip Roth, pour ne nommer que quelques exemples bien disparates), des journaux intimes, comme celui de Virginia Woolf, des ouvrages autobiographiques, comme ceux de Colette et de Nathalie Sarraute, des correspondances, ainsi que des ouvrages historiques et philosophiques - comme ceux de Saul Bellow, Henri Bergson, Hannah Arendt et Simone Weil, des exemples qui donnent tout juste un aperçu de l'étendue de ses lectures. Même si chaque texte dont discute Bosco ne porte pas directement sur la Shoah ou la Seconde Guerre mondiale, cellesci ne s'éloignent jamais de sa réflexion. Si Bosco se plonge dans une sélection de divers genres, auteurs et textes, elle trouve aussi que le hasard fait souvent bien les choses, tombant parfois sur des textes qui semblent avoir été «écrits pour moi, juste pour m'aider à franchir certains passages, à me tenir en haleine quand je sens que je vais peut-être perdre pied. $»^{26}$ Alors que certains auteurs sont évoqués de façon passagère, il y en a d'autres qui font l'objet de lectures soutenues et fréquentes.

Tel est le cas, entre autres, pour Virginia Woolf et Colette. Écrivaines que Bosco avoue aimer comme des «grandes sœurs, » elles étaient toutes deux mariées à des hommes juifs, et la montée du nazisme a eu un impact sur chacune d'entre elles. ${ }^{27} \mathrm{En}$ décrivant le journal intime de Woolf comme un « journal de guerre, » Bosco s'attarde au pacte de suicide entre Virginia et Léonard, la solution que propose Leonard au cas où l'Angleterre serait envahie par l'Allemagne.$^{28} \mathrm{Car}$, à son avis, si l'Angleterre devait capituler, cela signifierait, comme l'écrit Virginia dans son journal, «l'abandon de tous les Juifs. Les camps de concentration. $»^{29}$ Si Virginia exprime sa réticence à propos de ce pacte, n'ayant nulle «envie que [sa] vie ne se termine dans ce garage, $»^{30}$ ce qui interpelle davantage Bosco c'est le fait que Leonard ne se faisait aucune illusion sur le sort réservé aux Juifs pendant la Guerre dès i940. Bosco écrit :

Je m'étonne encore aujourd'hui de cette prescience de Leonard Woolf sur ce qui attendrait les Juifs sous l'occupation allemande. Certes, l'Angleterre n'eut pas à la subir, mais ce sont tous ceux du continent qui l'apprirent. Et bien plus tard. Sans trop vouloir le croire, un peu partout en Europe. Comment, en 1940, Leonard Woolf savait-il que le seul refuge à sa portée demeurait son garage ? $^{3}$ 
La lecture du journal de Virginia Woolf met en lumière pour Bosco les craintes et l'anxiété éprouvées par cette première au début de la Guerre, alors que Bosco était à peine au seuil de l'adolescence au début des années 1940. Mais encore plus que la guerre et la mort qui sont dépeintes par Woolf, c'est aussi son portrait lucide et sensible de la condition humaine qui émeut Bosco.

Contemporaine de Woolf, Colette prend pour troisième mari un homme juif, Maurice Goudeket, qui a été envoyé à Compiègne, où il aurait dû être ensuite déporté à Auschwitz si elle n'avait pas remué «mer et monde pour le faire libérer, $\aleph^{32}$ phrase qui sera d'ailleurs répétée presque mot pour mot dans Mea culpa.$^{33}$ Elle cite un long passage de L'Étoile Vesper, publié en 1946, ${ }^{34}$ qu'elle analyse assidument. Bosco souligne le fait que Colette n'ose parler explicitement des expériences de son mari dans ses écrits autobiographiques, constatant que celle-ci «est encore terriblement sur ses gardes [...] et continue d'être prudente, $»^{35}$ même après la libération de la France et la fin de la Guerre. Dans Mea culpa, paru trois ans avant Eh bien! la guerre, Bosco observe au sujet de Colette qu'elle ne dit rien ou du moins, n'écrit rien à propos de «toutes les horreurs de la guerre, des persécutions [...] Silence nécessaire sans doute. $\aleph^{36}$ Cette observation sur le silence nécessaire, voire imposé et inéluctable, n'est pas très éloignée de la réserve que l'on ressent chez Bosco, qui glisse à la surface de ses propres expériences de la Guerre, des années vécues cachée et la disparition des siens, que ce soit dans ses textes de fiction ou de non-fiction, avec retenue et effacement. Ces lectures de Colette, évoquées à plusieurs reprises dans divers essais, permettent à Bosco de mieux saisir pourquoi il était si difficile pour les rescapés des camps de concentration «à se faire entendre. ${ }^{37}$ On sait déjà dans quelle mesure il était presque impossible de mettre en mots l'horreur des expériences vécues, mais ce qui est encore plus inquiétant, selon Bosco, c'est le fait que même ceux qui ont réussi malgré tout à coucher sur papier leur témoignage, tels Robert Antelme ou Primo Lévi, ils n'ont pas été lus, car « on ne [voulait] rien entendre $»^{3^{8}}$ et ils « tombèrent dans un quasi-oubli, » constate-t-elle. ${ }^{39}$ Elle avoue qu'elle-même a mis «bien longtemps à les découvrir, ${ }^{{ }^{4}}$ mais qu'il aurait été important de les lire et qu'il ne soit d'ailleurs jamais trop tard pour le faire.

C'est le récit de Nathalie Sarraute qui retient davantage l'attention de Bosco, car même si ces deux femmes ne sont pas de la même génération, Sarraute ayant plutôt l'âge de la mère de Bosco, celle-ci se reconnait dans le vécu de son aînée. L'autrice naturalisée française, juive et d'origine russe, dont les parents, comme ceux de Bosco, ont divorcé lorsqu'elle était petite, a aussi vécu les années de guerre cachée et ne parlera que très peu de cette expérience que ce soit dans Enfance ou ses autres écrits. ${ }^{41}$ Si Bosco était réticente à lire l'autobiographie de Sarraute, elle avoue que c'est parce qu'elle craignait justement trop s'y retrouver. Sans approfondir la question, Bosco laisse entendre que son enfance n'a pas été facile et cela, bien avant la guerre. Comme Sarraute, elle a dû apprendre à s'intégrer dans le milieu scolaire français au point 
où elle a mis au rencart sa langue maternelle, l'allemand, langue qui rendait son intégration difficile en France, dans la période entre-deux-guerres et qui suscitait la méfiance..$^{42}$ Mais en lisant Sarraute, Bosco se laisse emporter dans les méandres de sa propre mémoire, évoquant des souvenirs d'école et ses « vertus assimilatrices, » du séjour en Bretagne au début de la guerre et du départ plus définitif vers Marseille, sans par contre oser creuser la mémoire afin de ramener à la surface ce qui a été, de son propre aveu, refoulé. ${ }^{43}$ Bosco admet qu'il y a des limites qu'elle n'est pas prête à franchir même si elle juge que les lectures et les écritures autobiographiques sont importantes afin de mieux (se) comprendre les impulsions, les actes, les refus, mais aussi les blessures qui bien que colmatées, ne sont pas guéries.

\section{Mémoire et oubli}

Dans ses essais, Bosco se reproche d'avoir trop oublié et de n'avoir pas su retenir les récits ou les noms de membres de sa famille qui ont disparu pendant la Shoah. Paradoxalement, elle affirme du même coup n'avoir rien oublié du tout. Dès le début de son dernier essai, Ces gens-là, elle révèle que même s'il y a des noms, des dates et d'autres détails qui lui échappent, les blessures de la guerre sont toujours à la surface et ne pourront jamais être oubliées. Il n'y a aucun effort à fournir pour se rappeler «la nuée de malédictions et fléaux» apportée par la guerre. ${ }^{44}$ Or, ce n'est pas faute d'avoir essayé de refouler cette mémoire : Bosco ne voulait pas se rappeler de tout ce qui l'a fait souffrir. «Pendant longtemps, ce fut l'oubli qu'on rechercha. L'oubli, le silence. Tout effacer de cette terreur, cette horreur que connurent nos pères et nos aïeux. $\gg^{45} \mathrm{La}$ gamme d'émotions qui entourent les souvenirs liés à la guerre est complexe, ce qui n'a pas de quoi surprendre. La crainte d'oublier, et son contraire, de se souvenir; la peur de sombrer dans la fureur et la peur tout simplement, toutes ces émotions hantent Bosco, mais, toujours confiante que la lecture peut répondre à ses interrogations, elle se tourne vers ses livres. C'est donc le livre d'Emmanuel Kattan, Penser le devoir de mémoire, qui incitera Bosco à réfléchir davantage à la problématique du devoir de mémoire, mais « de l'oubli aussi. $»^{46}$ Signalons d'ailleurs que dans cet ouvrage, Kattan consacre une large part de sa discussion aux fonctions de la mémoire dans la société et notamment au devoir de mémoire concernant la Shoah, où il souligne en outre la complexité des rapports entre l'impératif de la mémoire et de l'oubli. ${ }^{47}$

Si cette tension entre mémoire et oubli est omniprésente dans les essais de Bosco, force est de constater que la teneur de ses souvenirs n'est que rarement explicitée. Il est évident qu'il s'agit de souvenirs liés à son enfance, d'abord à Vienne puis à Paris, et plus tard, des années de guerre passées à Marseille. En évoquant l'impossibilité d'aller à l'école à cause d'un certificat de naissance qui ne pouvait pas être montré aux autorités «en ces années du "statut des Juifs" de Vichy,» Bosco éprouve de la difficulté à lire le livre de Jean Daniel, La prison juive. ${ }^{48}$ Elle s'y efforce pourtant, car, bien malgré 
elle, elle doit faire face à ce que constitue pour elle, cette «prison juive» : «On n'en finit jamais d'apprendre, à nos dépens, bien souvent, à quel point "la prison juive" est étanche, car les barreaux sont ainsi entrelacés qu'on ne peut jamais se faufiler à l'extérieur. $\gg^{49}$ Cette prison dans laquelle vit Bosco l'empêche d'oublier : sa culpabilité d'être juive, d'avoir survécu, d'avoir honte. En lisant La France et les Juifs, de I789 à nos jours de Michel Winock, Bosco poursuit le questionnement, en essayant de comprendre ce qui la «motivait à refouler le passé » ainsi que la peur qui la hantait..$^{50}$ Les chapitres traitant de Vichy et de l'Occupation de la France la bouleversent profondément, car elle réalise que, bien plus que la peur, c'est la rage refoulée, qui l'empêche en effet de voir, de comprendre et même de résister.

Ne pas voir, ne pas comprendre, ne rien entendre, ne rien dire.

Et la guerre finie, le danger écarté, on a continué à se taire - car la vérité était trop ignoble.

Pourquoi devrait-on accepter cette lâcheté qui rendait aveugle? Moi qui me suis tant lamentée sur mes trous de mémoire, je m’aperçois qu'au contraire je n'ai rien oublié du tout et je retrouve, malgré le temps perdu, ce temps sinistre, comme s'il était d'hier, là, toujours présent, ineffaçable. [...]

La seule vraie résistance permise était de survivre, de demeurer stoïque et de ne pas renoncer à «témoigner». Je me souviens encore du banc à Marseille où, subitement, je me jurai d'écrire tout cela. Oui, j'écrirais, je raconterais, je n'oublierais rien de ce que furent les compromissions, les lâchetés, les trahisons de l'époque. ${ }^{51}$

C'est d'ailleurs le désir de résister à l'oubli, à l'enfermement, à la culpabilité inexplicable et injustifiée, qui motive Bosco à écrire.

Or, lécriture ne se fait pas sans la lecture, dont la valeur est inestimable afin de pouvoir déceler les « compromissions, les lâchetés, les trahisons de l'époque, ${ }^{5^{2}}$ mais aussi les avertissements, les mises en garde, les idéologies, qui, si on y avait prêté une plus grande attention, auraient permis d'éviter le pire, ou au moins, de limiter le pouvoir de Hitler et le nombre de victimes. On ne lit jamais « assez de livres au moment où il le faudrait afin d'éviter d'autres désastres, car il y a toujours des Bérézina qui nous attendent. „53 En lisant un article notamment sur le philosophe Henri Bergson, qui avait prédit le sort réservé aux Juifs, Bosco affirme que cette lecture lui fait comprendre subitement ce qu'elle savait peut-être déjà depuis longtemps : «[...] les réponses se trouvaient dans les livres. $\aleph^{54}$ Elle évoque plus d'une fois aussi un essai de Nathalie Sarraute, «De Dostoïevski à Kafka " ${ }^{55}$ dans lequel celle-ci s’étonne de la perspicacité de Kafka qui, de son côté, avait prédit l'imposition du port de l'étoile jaune..$^{56}$ 
Simone Weil qui a «tout lu et surtout compris avec une précocité étonnante $\$^{57}$ impressionne Bosco et elle se reconnait dans la préoccupation de Weil, comme de Gertrude Stein d'ailleurs, pour le sort de tous ceux qui sont exclus, marginalisés, écartés de la société, prenant la défense de ceux qui n'ont pas de voix. Mais comme Stein, Weil ne prend pas la parole pour les groupes auxquels elle appartient, notamment les femmes et les Juifs. Contrairement à Bergson ou Kafka, Weil n'a pas pris pleinement conscience, selon Bosco, de ce qui attendait les Juifs à l'aube de la Seconde Guerre. «Elle [Weil] prend la parole avec courage, mais jamais en tant que femme, encore moins, bien évidemment, en tant que juive. Elle partage d'ailleurs cet étrange aveuglement avec Gertrude Stein, qui se refuse aussi à voir ce qui se passe durant ces années $40 . ~^{5^{8}}$ Pour Weil, il est incompréhensible que ce soit des lois qui définissent la judaité, alors qu'elle se réclame d'une «tradition chrétienne, française, hellénique. »59 Néanmoins, peu importe les traditions, l'éducation et la culture, il n'est pas possible d'échapper à cette «prison juive » évoquée plus tôt. Car, comme le constate Bosco, en lisant aussi un essai de Hannah Arendt, ${ }^{60}$ le paradoxe réside dans l'entre-deux, «être ou ne pas [...] être» juif, s'assimiler ou ne pas s'assimiler, peu importe car les deux positions suscitent la méfiance.

\section{Hitler, l'incitable, et Chaplin, le perspicace}

Le recours aux textes pour tenter de mieux comprendre les paradoxes liés à la judaïté, la méfiance envers les Juifs et finalement la Shoah atteint son paroxysme lorsque Bosco se contraint à lire, voire à relire, «avec répugnance [...] le fameux livre d'Hitler. ${ }^{6}{ }^{6}$ C'est contre sa propre volonté que Bosco se force à lire ce texte odieux, une lecture qu'elle juge néanmoins essentielle «pour vraiment savoir si tous les poisons $\mathrm{du}$ racisme et de la haine sont toujours aussi virulents. Juste d'en feuilleter quelques pages me rend malade. ${ }^{62}$ Par un effet de contraste, l'objectif de Bosco - qui est de comprendre comment cette horreur inexprimable qu'est la Shoah ait pu se produire - est mis en évidence par le refus catégorique de Nathalie Sarraute d'essayer de comprendre. "Oui, je comprends Sarraute et son "désir de ne-pas-comprendre définitif et total." Elle a humainement raison, sagement raison. Il y a des mots et des phrases qui ne doivent pas franchir certains barrages. ${ }^{63}$ Pourtant, elle poursuit sa lecture malgré la profonde répulsion qu'elle ressent Elle s'engage toutefois à ne pas honorer ce livre en le citant, mais affirme qu'il est important de le confronter afin de voir s'il aurait été possible, si on l'avait lu dans les années 1930, de percevoir non seulement la haine, mais aussi les plans de domination et d'annihilation que Hitler fomentait. Elle ne tiendra pas sa promesse, par contre, car ce n'est qu'en citant les paroles mêmes de Hitler qu'elle arrive à montrer «la folie meurtrière, la haine du vaincu envers ses vainqueurs et surtout l'idée fixe du bouc émissaire, le Juif coupable de tout, capable de tout. ${ }^{64}$ Malgré le dégoût qu'elle ressent en faisant cette lecture, Bosco se garde néanmoins de revendiquer sa censure. Au contraire, ce n'est que par la lecture de Mein Kampf qu'il aurait été possible de changer le cours de l'histoire. Texte où la 
haine est omniprésente, il révèle non seulement l'ambition politique de Hitler, mais aussi le fanatisme et l'aveuglement provoqués par la haine.

Personnellement, je crois que, loin d'être interdite, la diffusion aurait pu et dû être encouragée. Alors, on aurait su ce qui nous attendait, ce qui nous attendrait, sans faute, si Hitler s'emparait du pouvoir. Mais on ne voulait sans doute rien savoir. C'est tellement plus facile de se fourrer la tête dans le sable. Dieu voulut peut-être perdre ceux qu'il avait autrefois choisis? On n'ose pas l'écrire et je ne veux pas le croire. Et j'essaie de poursuivre ma lecture même si elle est pénible. ${ }^{65}$

Cette citation présente un condensé des éléments qui incarnent les lamentations de Bosco. À l'instar de l'auteur du Livre des Lamentations, Bosco se demande pourquoi Dieu voulait détruire le peuple juif. Par ailleurs, elle fait un lien sans équivoque entre la lecture et la possibilité de comprendre ce qui motivait Hitler. Elle renchérit : «Il n'y avait qu'à le [Hitler] lire - tout simplement le lire avec attention [...] car il suffisait de constater qu'il était atteint de haine, de la haine la plus virulente qui soit, celle qui ne s'encombre de rien pour pouvoir satisfaire ce besoin de vengeance, une vengeance qui ne comptera pas les millions de morts nécessaires pour le satisfaire. ${ }^{66}$

Celui qui avait compris, étonnamment, c'était Charlie Chaplin. Mettant de côté les écrits haineux de Hitler, Bosco se tourne vers les films du comédien, notamment $L e$ Dictateur, présenté en version restaurée en salle de cinéma à Montréal, ainsi que son autobiographie, Histoire de ma vie. ${ }^{67}$ Ce qu'elle remarque tout particulièrement en la lisant, c'est que Charlie Chaplin avait compris, lui, la haine qui dominait Hitler et plus vite, d'ailleurs que «les autres, politiciens et généraux » de l'époque avant la guerre. ${ }^{68}$ Si Chaplin avoue dans son autobiographie qu'il n'était pas un grand lecteur et, selon Bosco, rien ne laisse croire que Chaplin aurait lu Mein Kampf, elle affirme tout de même qu'il a «su voir et comprendre avant les autres, voir et montrer l'exode des Juifs vers des trains qui ne mèneront qu'à la mort. ${ }^{69}$ En citant cette autobiographie assez longuement, Bosco souligne que Chaplin possédait une sensibilité et une perspicacité hors de commun. Elle cite Chaplin : «Si j'avais connu les réelles horreurs des camps de concentration allemands, je n'aurais pas pu réaliser Le Dictateur; je n'aurais pas pu tourner en dérision la folie homicide des nazis. Mais jétais décidé à ridiculiser leur bla-bla mystique sur les races au sang pur. . $7^{\circ}$

La Shoah et les textes qui l'évoquent - que ce soit les écrits de Hitler bien avant la Seconde Guerre mondiale ou les romans, essais, biographies publiées depuis - rejoignent la lamentation dans le sens où l'on pleure les morts, les blessés, les disparus, ceux qui ne sont jamais nés. Mais là n'est pas la ligne d'arrivée des Lamentations, ni, finalement, des essais de Bosco. Au-delà de la complainte et de l'écorchement, il faut poser des questions et tenter de comprendre. Peut-être faut-il du temps avant de pouvoir entreprendre cette quête et accepter aussi le risque de ne pas trouver les 
réponses que l'on cherche, ou encore de ne pas être satisfait par ce que l'on découvre. Mais pour Bosco, toute son entreprise littéraire - œuvres de fiction, de poésie, de non-fiction - participe à cette interrogation, à cet effort, cette quête, de compréhension. Ce qui est particulièrement remarquable dans les essais publiés au cours des dix dernières années de sa vie, c'est le recours insatiable aux textes des autres : écrivains, philosophes, historiens, journalistes et même un dictateur, pour faire la lumière sur l'épisode qui aura clivé le vingtième siècle, un peuple et le monde occidental.

Si la littérature demeure pertinente en temps de guerre et de paix, c'est elle seule qui porte «les marques, les stigmates, les cicatrices des horreurs d'hier et de celles qui s'annonc[ent] pour demain. $»^{71} \mathrm{Il}$ importe de signaler que si la Shoah et la Seconde Guerre mondiale sont au cœur des préoccupations de Bosco, elles sont toujours mises en contexte avec d'autres événements mondiaux, tels que le génocide arménien, la Guerre du Vietnam, la dictature en Haïti et de surcroit, la seconde guerre en Irak qui se préparait lorsqu'elle écrivait Eh bien! la guerre et qui venait d'éclater au moment de l'écriture de Ces gens-là, mais aussi, aux conflits possibles, ceux qui pourraient survenir. Comprendre les guerres, les massacres, les abus de pouvoir et les autres fléaux font partie des responsabilités de tout un chacun, même des littéraires, non seulement pour sauvegarder le passé, mais aussi, l'avenir. Il est clair que pour elle, il s'agit de continuer à lire et à reconnaitre, dans ce qu'on lit, les enseignements sur et pour l'humanité.

1

Monique Bosco, Confiteor (Montréal : Hurtubise $\mathrm{HMH}, 1998), 36$.

\section{2}

André Brochu, « Portrait de Minerve peint par elle-même, entrevue avec Monique Bosco, » Voix et images, dossier consacré à Monique Bosco, 9, no. 3 (1984) : 9.

3

Monique Bosco, Eh bien! la guerre (Montréal : Hurtubise HMH, 2004), 100.

\section{4}

Jean-Marc Droin, Le Livre des Lamentations. "Comment? » Une traduction et un commentaire (Genève : Labor et Fides, 1995), 10.

5

Tod Linafelt, Surviving Lamentations: $\mathrm{Ca}$ tastrophe, Lament and Protest in the Afterlife of a Biblical Book (Chicago: University of Chicago Press, 2000), 23-24.

\section{6}

Monique Bosco, Babel-Opéra (Laval : Quinze, 1989), 13-14.

\section{7}

Leora Batnitzsky, Preface in Lament in Jewish Thought: Philosophical, Theological, and Literary Perspectives, ed. Ilit Ferber and Paula Schwebel, Perspectives on Jewish Texts and Contexts 2 (Berlin / Boston: De Gruyter, 2014), xiii.

\section{8}

Georges Didi-Huberman, Ninfa dolorasa : Essai sur la mémoire d'un geste (Paris : Gallimard, 2019), 260-61. Les italiques sont dans le texte.

9

Monique Bosco, Ces gens-là (Montréal : Hurtubise $\mathrm{HMH}, 2006$ ). 
10

Nancy Arenberg, "Fossilized Memories and Loss in Monique Bosco's La femme de Loth," Québec Studies 59 (2015): 136-37. Ma traduction.

\section{1}

Voici l'exergue :

« Je suis devant le mur

Le long mur

Le mur des lamentations

Le mur des ancêtres qui,

La face couverte de cendres,

Venaient pour s'y lamenter

Se lamenter

La seule chose gratuite

Suprême consolation permise. »

Monique Bosco, Un amour maladroit (Paris :

Gallimard, 1961), 7.

\section{2}

Michael Greenstein, Third Solitudes: Tradition and Discontinuity in Jewish-Canadian Literature (Kingston / Montreal: McGill-Queen's University Press, 1989), 162.

\section{3}

Irène Oore, « Regards et reflets : du rejet de soi vers la réconciliation dans Un amour maladroit de Monique Bosco, » dans Entre textes et images : constructions identitaires en Acadie et au Québec, dir. Monika Boehringer, Kirsty Bell et Hans R. Runte (Moncton: Université de Moncton, 2010), 256.

\section{4}

Oore, 256.

\section{5}

Monique Bosco, « Les lamentations de la vieille en ce jour du Kippour, » Boomerang (Montréal : Hurtubise HMH, 1987), 73-84.

\section{6}

Cristina Minelle, « Monique Bosco: Les lamentations de la vieille en ce jour du Kippour, » Romanica Silesiana 2 (2007) : 50-58.

\section{7}

Voir, à titre d'exemples : Arenberg, "Fossilized Memories"; Marie Carrière, Médée protéiforme (Ottawa : Les Presses de l'Université d'Ottawa, 2012); Gloria Escomel, « Monique Bosco ou le miroir brisé, » La Nouvelle Barre du jour 65 (1978) : 90-97; Greenstein, Third Solitudes; Catherine Khordoc, «La tour de lamentations : Babel-Opéra de Monique Bosco, » dans Tours et détours : le mythe de Babel dans la littérature contemporaine, par Khordoc (Ottawa : Presses de l'Université d'Ottawa, 2012), 161-91; Pierre L'Hérault, «Les mythologies de Monique Bosco, » dans Multi-culture, multi-écriture. La voix migrante au féminin en France et au Canada, dir. Lucie Lequin et Maïr Verthuy, Critiques littéraires (Paris/Montréal : L'Harmattan, 1996), 59-71; Minelle, « Monique Bosco : les lamentations »; et Oore, « Regards et reflets. »

\section{8}

Bosco, Confiteor, 86.

19

Bosco, Ces gens-là, 102.

\section{0}

Bosco, Eh bien!, 73.

\section{1}

Bosco, Eh bien!, 66.

\section{2}

Voir Catherine Khordoc, « 'Si tu t'imagines, fillette, fillette, si tu t'imagines' : refrains et autres répétitions dans l'œuvre de Monique Bosco, » dans La répétition dans les textes littéraires du Moyen-Âge à nos jours, dir. Loula Abd-elrazak et Valérie Dusaillant-Fernandes (New York : Peter Lang, 2020), 193-205.

\section{3}

Élisabeth Nardout-Lafarge, « L'émergence de la voix essayistique dans les textes de Monique Bosco, » dans Parcours de l'essai québécois (1980-2000), dir. Anne Caumartin et Martine-Emmanuelle Lapointe (Québec : Nota bene, 2004), 37.

\section{4}

La voix auctoriale du Livre des Lamentations a longtemps été attribuée à Jérémie, mais de nombreux exégètes rejettent cette théorie. Voir Droin, pp. 16-17.

\section{5}

Voir à cet égard Catherine Khordoc, « 'Éclipse de Dieu' : allusions et contestations bibliques dans l'œuvre de Monique Bosco, "Studies in Canadian Literature / Études en littérature canadienne 42, no. 2 (2017): 48-65; Hans Jonas, Le concept de Dieu après Auschwitz : une voix juive, trad. Philippe Ivernel (Paris : Payot et Rivages, 1994). 
26

Bosco, Ces gens-là, 47.

27

Bosco, Eh bien!, 40.

28

Bosco, Eh bien!, 43.

29

Bosco, Eh bien!, 44. Les italiques sont dans le texte.

\section{0}

Bosco fait remarquer que Virginia Woolf se suicide quelques semaines après qu'elle ait exprimé, dans son journal, son désaccord avec le pacte de suicide de son mari, car elle affirme souhaiter vivre « encore dix ans afin de pouvoir écrire le livre qui comme à l'accoutumée afflue dans ma tête. " Bosco, Eh bien!, 44.

\section{1}

Bosco, Eh bien!, 44.

\section{2}

Bosco, Eh bien!, 41.

\section{3}

Monique Bosco, Mea culpa (Montréal : Hurtubise HMH, 2001), 91.

\section{4}

Bosco cite le IVe tome de l'œuvre complète de Colette publiée dans la collection « Pléiade. »

\section{5}

Bosco, Eh bien!, 58.

\section{6}

Bosco, Mea culpa, 91.

\section{7}

Bosco, Eh bien!, 58.

\section{8}

Monique Bosco, Bis (Montréal : Hurtubise HMH, 1999), 80.

\section{9}

Bosco, Eh bien!, 59.

\section{0}

Bosco, Confiteor, 81-82.

\section{1}

Bosco, Bis, 80.

\section{2}

Bosco, Bis, 92.

\section{3}

Bosco, Bis, 92-94.

44

Bosco, Ces gens-là, 8 .

\section{5}

Bosco, Ces gens-là, 51 .

\section{6}

Bosco, Ces gens-là, 52 .

47

Emmanuel Kattan, Penser le devoir de mémoire (Paris : Presses universitaires de France, 2002).

\section{8}

Bosco, Ces gens-là, 79 .

49

Bosco, Ces gens-là, 80 .

50

Bosco, Ces gens-là, 163.

51

Bosco, Ces gens-là, 163-64.

52

Bosco, Ces gens-là, 164.

53

Bosco, Ces gens-là, 70 .

54

Bosco, Eh bien!, 66.

55

Nathalie Sarraute, « De Dostoïevski à Kafka, » Les Temps modernes (octobre 1947).

56

Bosco, Bis, 79; Eh bien!,60.

57

Bosco, Eh bien!, 72.

58

Bosco, Eh bien!, 84.

59

Bosco, Eh bien!, 78. Bosco cite Weil. 
60

Hannah Arendt, Sur l'antisémitisme (Paris :

Seuil, 1998).

61

Bosco, Eh bien!, 66 .

62

Bosco, Eh bien!, 66 .

63

Bosco, Eh bien!, 67.

64

Bosco, Eh bien!, 117-18.

65

Bosco, Eh bien!, 111.

66

Bosco, Eh bien!, 118.

67

Charles Chaplin, Histoire de ma vie, trad. Jean Rosenthal (Paris : Robert Laffont, 1964).

68

Bosco, Eh bien!, 139.

69

Bosco, Eh bien!, 139.

70

Bosco, Eh bien!, 140. Les italiques sont dans le texte.

71

Bosco, Eh bien!, 17. 